\title{
Troponina I en pacientes con tromboembolismo pulmonar
}

J. M. CALVO ROMERO

Medicina Interna. Hospital de Zafra. Zafra. Badajoz
TROPONIN I IN PATIENTS WITH PULMONARY THROMBOEMBOLISM

\section{RESUMEN}

Objetivo: Estudiar la frecuencia en pacientes con tromboembolismo pulmonar (TEP) de una troponina I elevada y las características asociadas.

Material y métodos: Estudio prospectivo de 29 pacientes consecutivos con TEP.

Resultados: La troponina I fue determinada en 22 pacientes. La edad media fue 78,1 años. Diez $(45,5 \%)$ tuvieron una troponina I elevada, con un nivel medio de $1 \mathrm{ng} / \mathrm{ml}$ (rango 0,7-3,1 ng/ml). El retraso diagnóstico fue menor en los pacientes con troponina I elevada $(2 \pm 1,7$ vs. 5,9 $\pm 5,6$ días, $\mathrm{p}=0,04)$. Tres $(30 \%)$ de los 10 pacientes con troponina I elevada presentaron dolor centrotorácico. Los pacientes con troponina I elevada tuvieron una mayor frecuencia de hipotensión arterial (40 vs. 16,7\%), insuficiencia respiratoria $(70 \%$ vs. $58,3 \%)$ y datos electrocardiográficos de sobrecarga derecha (50 vs. 33,3\%), aunque sin alcanzar significación estadística.

Conclusiones: Una troponina I ligeramente elevada es frecuente en pacientes con TEP. El diagnóstico precoz puede aumentar la frecuencia de una troponina I elevada. La coexistencia de una troponina I elevada y dolor centrotorácico puede simular un síndrome coronario agudo. En pacientes con TEP, una troponina I elevada puede asociarse a algunos criterios de gravedad como hipotensión arterial, insuficiencia respiratoria y datos electrocardiográficos de sobrecarga derecha.

PALABRAS CLAVE: Troponina. Troponina I. Tromboembolismo pulmonar. Embolismo pulmonar.

\section{ABSTRACT}

Objective: To study the frequency in patients with pulmonary thromboembolism (PTE) of an elevated troponin I and the associated characteristics.

Material and methods: Prospective study of 29 consecutive patients with PTE.

Results: Troponin I was determined in 22 patients. The median age was 78.1 years. Ten (45.5\%) had an elevated troponin I, with a median level of $1 \mathrm{ng} / \mathrm{ml}$ (range 0.7-3.1 $\mathrm{ng} / \mathrm{ml}$ ). The diagnostic delay was lower in patients with an elevated troponin $I(2 \pm 1.7 v s .5 .9 \pm 5.6$ days, $p=0.04)$. Three (30\%) of 10 patients with an elevated troponin I had midthoracic pain. Patients with an elevated troponin I had a higher frequency of arterial hypotension (40 vs. $16.7 \%)$, respiratory failure (70 vs. 58.3\%) and electrocardiographic overload right heart data (50\% vs. 33.3\%), although without reaching statistical significance.

Conclusions: A slightly elevated troponin I is frequent in patients with PTE. The early diagnosis may increase the frequency of an elevated troponin I. The coexistence of an elevated troponin I and midthoracic pain may simulate an acute coronary syndrome. In patients with PTE, an elevated troponin I may associate to some severity criteria such as arterial hypotension, respiratory failure and electrocardiographic overload right heart data.

KEY WORDS: Troponin. Troponin I. Pulmonary thromboembolism. Pulmonary embolism.

Calvo Romero JM. Troponina I en pacientes con tromboembolismo pulmonar. An Med Interna (Madrid) 2005; 22: 589-590.

\section{INTRODUCCIÓN}

La troponina I y la troponina $\mathrm{T}$ son los marcadores de laboratorio más sensibles y específicos de lesión miocárdica, y habitualmente su elevación indica la existencia de un síndrome coronario agudo (1). La troponina también puede elevarse en el tromboembolismo pulmonar (TEP), y se le ha atribuido un valor pronóstico (2-6). En el presente estudio, se describen la frecuencia en una serie de pacientes con TEP de una troponina I elevada y las características asociadas a esta elevación.

\section{CASOS APORTADOS}

Se realizó un estudio prospectivo de todos los pacientes diagnosticados de TEP entre noviembre de 2003 y diciembre de 2004 en un hospital de primer nivel de un área rural del sur de Extremadura. En $22(75,9 \%)$ de un total de 29 pacientes con TEP se determinó la troponina I en las primeras 24 horas del ingreso, con al menos 2 determinaciones separadas por 6-12 horas. La determinación de troponina I se realizó mediante un método de enzimo-inmunoensayo colorimétrico (CTNI Flex, sistema Dimension; Dade Behring). Se consideró elevada cuando el nivel fue superior a $0,6 \mathrm{ng} / \mathrm{ml}$ (límite establecido por el fabricante). El diagnóstico de TEP se estableció por una gam-

Trabajo aceptado: 23 de junio de 2005

Correspondencia: J. M. Calvo Romero. Sergio Luna, 15, 2ª. 06010 Badajoz. e-mail: jmcromero@eresmas.com 
magrafía pulmonar de ventilación/perfusión de alta probabilidad (11 pacientes), o por la asociación de un cuadro clínico compatible con TEP y la demostración de trombosis venosa profunda mediante ecografía-doppler venosa de miembros inferiores (11 pacientes).

Las variables continuas se expresan como media \pm desviación estándar. El análisis estadístico se realizó utilizando la prueba Chi cuadrado y el test exacto de Fisher, cuando alguno de los valores esperados fue menor de 5, para la comparación de proporciones, y la prueba de la " $t$ " de Student para la comparación de medias. Se consideró estadísticamente significativo un valor de $\mathrm{p}<0,05$.

La edad media de los 22 pacientes fue 78,1 años (rango 35-94 años), y $15(68,2 \%)$ fueron mujeres. La troponina I resultó elevada en 10 pacientes $(45,5 \%)$. La media de los niveles máximos de troponina I en los 10 pacientes con troponina I elevada fue $1 \mathrm{ng} / \mathrm{ml}$ (rango $0,7-3,1 \mathrm{ng} / \mathrm{ml})$. En un paciente $(4,5 \%)$ la troponina I fue superior a 2 $\mathrm{ng} / \mathrm{ml}$. En la tabla I se comparan las características de los pacientes con troponina I elevada y troponina I normal.

\section{DISCUSIÓN}

En un $31-47 \%$ de los pacientes con TEP se puede encontrar una elevación de la troponina I, que suele ser moderada (2-6). Estos resultados son similares a los de este estudio, en el que casi la mitad de los pacientes con TEP tuvieron una troponina I elevada y el nivel medio de troponina I en los pacientes con troponina I elevada fue $1 \mathrm{ng} / \mathrm{ml}$, con sólo un paciente con un nivel superior a $2 \mathrm{ng} / \mathrm{ml}$.

El retraso diagnóstico fue significativamente menor en los pacientes del estudio con una troponina I elevada, lo que sugiere que el diagnóstico precoz puede aumentar la frecuencia de una troponina I elevada en pacientes con TEP. En este sentido, se ha descrito que la cinética de liberación de la troponina $\mathrm{T}$ en los pacientes con TEP es distinta de la de los pacientes con síndrome coronario agudo, con un pico de troponina $\mathrm{T}$ aproximadamente a las 10 horas del inicio de los síntomas de TEP y niveles detectables sólo durante una media de 40 horas (7). La troponina puede elevarse en pacientes con insuficiencia renal crónica terminal (8). No obstante, la frecuencia de insuficiencia renal en los pacientes de esta serie con troponina I elevada y troponina I normal fue similar y de aproximadamente sólo un $10 \%$. También debe destacarse que
TABLA I

COMPARACIÓN DE LAS CARACTERÍSTICAS DE LOS PACIENTES CON TROPONINA I ELEVADA Y TROPONINA I NORMAL

\begin{tabular}{lccc}
\hline & $\begin{array}{c}\text { Troponina l elevada } \\
n=10\end{array}$ & $\begin{array}{c}\text { Troponina I normal } \\
n=12\end{array}$ & $p$ \\
\hline Edad & $80,8 \pm 7,6$ & $75,8 \pm 15,3$ & NS \\
Edad > 75 años & $7(70 \%)$ & $8(66,7 \%)$ & NS \\
Sexo femenino & $8(80 \%)$ & $7(58,3 \%)$ & NS \\
Retraso diagnóstico (días) & $2 \pm 1,7$ & $5,9 \pm 5,6$ & 0,04 \\
Dolor centrotorácico & $3(30 \%)$ & $4(33,3 \%)$ & NS \\
TAS 100 mmHg & $4(40 \%)$ & $2(16,7 \%)$ & NS \\
Insuficiencia respiratoria & $7(70 \%)$ & $7(58,3 \%)$ & NS \\
Sobrecarga derecha en ECG* & $5(50 \%)$ & $4(33,3 \%)$ & NS \\
Insuficiencia renal** & $1(10 \%)$ & $1(8,3 \%)$ & NS \\
Muerte intrahospitalaria & $1(10 \%)$ & 0 & NS \\
\hline TAS: tension arteia & & 0 &
\end{tabular}

TAS: tensión arterial sistólica al ingreso. ECG: electrocardiograma. ${ }^{*}$ Bloqueo de rama derecha, onda T negativa en V1-V3 y/o patrón S1Q3T3.

${ }^{*}$ Creatinina plasmática $>1,5 \mathrm{mg} / \mathrm{dl}$. NS: no significación estadística ( $p$ $\geq 0,05$ ).

casi un tercio de los pacientes con troponina I elevada tuvieron dolor centrotorácico, pudiendo simular un síndrome coronario agudo.

La troponina ha demostrado ser un predictor de mortalidad y de un curso complicado en pacientes con TEP (2-6). La troponina se eleva en el TEP probablemente debido a una sobrecarga aguda del corazón derecho, y se correlaciona con la presencia de disfunción ventricular derecha valorada mediante ecocardiografía (2-6). El número de muertes en este estudio (sólo una en el grupo con troponina I elevada) no permite sacar conclusiones sobre el valor de la troponina I como predictor de mortalidad. No obstante, una troponina I elevada en este estudio se asoció a una mayor frecuencia, aunque sin alcanzar significación estadística, de algunos criterios de gravedad como hipotensión arterial, insuficiencia respiratoria y datos electrocardiográficos de sobrecarga derecha.

\section{Bibliografía}

1. Panteghini M. Acute coronary syndrome: biochemical strategies in the troponin era. Chest 2002; 122: 1428-35.

2. Meyer T, Binder L, Hruska N, Luthe H, Buchwald AB. Cardiac troponin I elevation in acute pulmonary embolism is associated with right ventricular dysfunction. J Am Coll Cardiol 2000; 36: 1632-6.

3. Konstantinides S, Geibel A, Olschewski M, Kasper W, Hruska N, Jackle $\mathrm{S}$, et al. Importance of cardiac troponins I and T in risk stratification of patients with acute pulmonary embolism. Circulation 2002; 106: 1263-8.

4. Mehta NJ, Jani K, Khan IA. Clinical usefulness and prognostic value of elevated cardiac troponin I levels in acute pulmonary embolism. Am Heart J 2003; 145: 821-5.

5. Kucher N, Wallmann D, Carone A, Windecker S, Meier B, Hess OM.

Incremental prognostic value of troponin I and echocardiography in patients with acute pulmonary embolism. Eur Heart J 2003; 24: 16516.

6. La Vecchia L, Ottani F, Favero L, Spadaro GL, Rubboli A, Boanno C, et al. Increased cardiac troponin I on admission predicts in-hospital mortality in acute pulmonary embolism. Heart 2004; 90: 633-7.

7. Müller-Bardorff M, Weidtmann B, Giannitsis E, Kurowski V, Katus HA. Release kinetics of cardiac troponin $\mathrm{T}$ in survivors of confirmed severe pulmonary embolism. Clin Chem 2002; 48: 673-5.

8. Apple FS, Murakami MM, Pearce LA, Herzog CA. Predictive value of cardiac troponin I and $\mathrm{T}$ for subsequent death in end-stage renal disease. Circulation 2002; 106: 2941-5. 